\title{
El área de información y alfabetización informacional de la competencia digital docente
}

\author{
The Information and Information Literacy Area of the Digital Teaching Competence
}

\section{A área de informação e alfabetização informacional da competência digital do ensino}

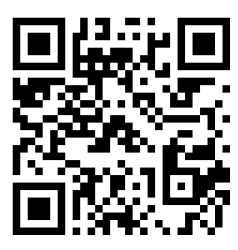

(iD) https://orcid.org/0000-0002-2210-9918

Ana Bueno-Pedrero Universidad de Granada Ceuta, España anabp@correo.ugr.es (iD) https://orcid.org/0000-0002-9641-961X

Laura Borrego-Otero Universidad de Granada

Ceuta, España lauraborregoo@correo.ugr.es

iD https://orcid.org/0000-0002-7789-7640

Recibido • Received • Recebido: 23 / 01 / 2019

Corregido • Revised $\cdot$ Revisado: 28 / 07 / 2020

Aceptado • Accepted $\cdot$ Aprovado: 18 / 08 / 2020

\begin{abstract}
Resumen: Las tecnologías de la información y comunicación (TIC) son parte integrante de nuestra vida. Este hecho se ve reflejado en el sistema educativo, donde el personal docente debe tener adquirida una serie de competencias digitales, necesarias para el desarrollo de esta misma en el estudiantado. El estudio trata de describir el área de competencia digital, información y alfabetización informacional, del estudiantado del Máster de Profesorado de Educación Secundaria Obligatoria, Bachillerato, Formación Profesional y Enseñanza de Idiomas en el Campus de Ceuta. Se ha empleado un método cuantitativo, con alcance descriptivo, mediante diseño no experimental, de paradigma longitudinal. La muestra es de 153 estudiantes. El instrumento utilizado es un cuestionario fundamentado en el Marco Común de Competencia Digital Docente de 2017, con escala tipo Likert de 6 puntos, y subdividido en tres bloques: A ( $\mathrm{A} 1$ y A2), considerado como nivel básico, B (B1 y B2), como nivel intermedio, y C (C1 y C2), como nivel avanzado. Los resultados muestran que los futuros grupos docentes tienen
\end{abstract}


http://doi.org/10.15359/ree.24-3.25

http://www.una.ac.cr/educare

educare@una.ac.cr

un nivel medio en el área competencia de información y alfabetización informacional, y presentan valores más altos en el almacenamiento y recuperación de información, datos y contenidos digitales; niveles medios en el almacenamiento y recuperación de información, de datos y contenidos digitales; y más bajos en la evaluación de información, datos y contenidos digitales. Podemos concluir que es necesaria una formación centrada en el desarrollo de las competencias digitales docentes, lo cual requiere, por parte de las autoridades competentes, de planes de formación adecuados.

Palabras claves: Docente; formación; competencias del personal docente; tecnología de la información.

\begin{abstract}
Information and Communication Technologies (ICT) are an integral part of our lives. This fact is reflected in the educational system, where teachers must have acquired a series of digital skills that should be developed in students. The study tries to describe the area of digital competence, information, and information literacy in the students of the Master's Degree in Compulsory Secondary Education, Baccalaureate, Vocational Training, and Language Teaching in the Campus of Ceuta. A quantitative method has been used; it has a descriptive scope and a non-experimental design of a longitudinal paradigm. The sample is 153 students. The instrument implemented is a questionnaire based on the Common Framework of Digital Teacher Competence of 2017, with a Likert scale of 6 points, and subdivided into three blocks: $A$ ( $A 1$ and A2), considered as a basic level, B (B1 and B2), as an intermediate level, and C (C1 and C2), as an advanced level. The results show that future teachers have a medium level in the area of information competence and information literacy, presenting higher values in the storage and retrieval of information, data, and digital content. They also have average levels in the storage and retrieval of information, data and digital content, and lower levels in evaluating information, data, and digital content. We can conclude that training centered on developing digital teaching competencies is necessary, requiring, on the part of the competent authorities, adequate training plans.
\end{abstract}

Keywords: Teachers; training; teacher qualifications; information technology.

Resumo: As Tecnologias da Comunicação e Informação (TIC) são parte integrante das nossas vidas. Este fato se reflete no sistema de ensino, onde os professores devem ter adquirido uma série de competências digitais necessárias para o desenvolvimento das mesmas nos estudantes. Esse estudo busca descrever a área de competência digital informação e alfabetização informacional dos estudantes do Mestrado em docência de educação básica, Bacharelado, Formação Profissional e Ensino de Língua no Campus de Ceuta. Foi utilizado um método quantitativo, descritivo através de uma proposta não experimental, com paradigma longitudinal. A amostra é de 153 estudantes. $O$ instrumento utilizado é um questionário baseado no quadro comum de Competência Digital Docente de 2017, com escala de Likert de 6 pontos, subdivididas em três blocos: A (A1 e A2), considerados como nível básico, B (B1 e B2) como nível médio, e C ( 1 e C2), como nível avançado. Os resultados mostram que os futuros professores têm um nível médio na área da competência de informação e alfabetização informacional, apresentando valores mais elevados no armazenamento e recuperação de informações, dados e conteúdos digitais; nível médio em armazenamento e recuperação de informação, de dados e conteúdo digital; e nível baixo na avaliação de informações, dados e conteúdo digital. Podemos concluir que é necessário um treinamento focado no desenvolvimento de habilidades digitais de ensino, exigindo, por parte das autoridades competentes, planos de treinamento adequados.

Palavras-chave: Professor; formação; competências de professores; tecnologia da informação. 
http://doi.org/10.15359/ree.24-3.25

http://www.una.ac.cr/educare educare@una.ac.cr

\section{Introducción}

El estudio que a continuación se presenta parte de un documento elaborado por el Instituto de Tecnologías Educativas y Formación del Profesorado, realizado en octubre de 2017, sobre el Marco común de competencia digital docente.

La intención principal de esta investigación es describir el área de competencia digital, información y alfabetización informacional, de estudiantes del Máster de Profesorado de Educación Secundaria Obligatoria, Bachillerato, Formación Profesional y Enseñanza de Idiomas en el Campus de Ceuta.

De manera más específica, se trata de identificar el nivel competencial progresivo del estudiante en la navegación, búsqueda y filtrado de información, datos y contenidos digitales; evaluación de información, datos y contenidos digitales; y en el de almacenamiento y recuperación de información, datos y contenidos digitales de dicho alumnado.

Las tecnologías de la información y comunicación han tenido un crecimiento exponencial en los últimos tiempos en el ámbito social (Cabezas González et al., 2014) y educativo (Moreno Guerrero y López Belmonte, 2018; Trujillo Torres y Raso Sánchez, 2010), y han generado una revolución digital en la formación estudiantil (Cózar Gutiérrez y Roblizo Colmenero, 2014). Ello ha provocado movimientos, por parte de las instituciones gubernamentales, que tratan de aplicar políticas educativas centradas en la formación inicial y continua de los grupos de discentes (Sánchez-Antolín et al., 2014), sobre todo en la educación superior (Agreda Montoro et al., 2016).

Las personas responsables de la educación del estudiantado deben ser competentes en el uso de las tecnologías de la información y comunicación (Cabezas González et al., 2014). Ello hace que uno de los requisitos del equipo de docentes de hoy en día es ser competente digitalmente (Cózar Gutiérrez y Roblizo Colmenero, 2014), con destrezas y conocimientos en el uso de las TIC en muchas vertientes (Gisbert Cervera et al., 2016), en la que destaca el acceso a la información presentada de manera colaborativa en la red (Trujillo Torres y Raso Sánchez, 2010).

Esto que hemos comentado ahora mismo es lo ideal, pero la realidad que encontramos en nuestras aulas es distinta, dado que la formación que presenta el personal docente y la que transmite al estudiantado, en lo que a competencia digital se refiere, es deficiente (Cabezas González et al., 2014).

¿Cuáles son los motivos de la escasa formación académica y pedagógica en competencia digital del personal docente? Los estudios centran la problemática en lo inadecuada de esta, la cual está muy instrumentalizada (Fernández Martín et al., 2002; Valverde Berrocoso, 2015). Esto se ve reflejado en procesos de enseñanza y aprendizaje con poca o nula integración de las TIC (Cózar Gutiérrez y Roblizo Colmenero, 2014). En realidad, el docente y la docente con una competencia digital adecuada suelen aplicar metodologías acordes al uso de las TIC, con dinámicas activas (Esteve Mon et al., 2014) y presentan una formación tanto tecnológica como pedagógica (Gisbert Cervera et al., 2016). Esto genera cambios en el rol docente, el cual pasa de 
http://doi.org/10.15359/ree.24-3.25

http://www.una.ac.cr/educare

educare@una.ac.cr

una función de transmisión de conocimientos, mediante la aplicación del método expositivo, a convertirse en guía del proceso de enseñanza y aprendizaje del alumnado, mediante el desarrollo de métodos pedagógicos de corte socioconstructivista (Gisbert Cervera et al., 2016).

El uso de las TIC en los procesos de enseñanza y aprendizaje no debe centrarse en el análisis descriptivo de las potencialidades educativas de los recursos tecnológicos en sí, sino, más bien, en establecer una base pedagógica (Esteve-Mon et al., 2016) y transversal (Gisbert Cervera et al., 2016). Es decir, los recursos tecnológicos aplicados en el ámbito educativo deben ser analizados, de forma conjunta, con las metodologías aplicadas.

Los futuros grupos docentes, en su conjunto, aun siendo nativos digitales, presentan una formación centrada en el ocio y la comunicación personal, si se tienen en cuenta estudios aplicados con anterioridad (Cantón-Mayo et al., 2017); queda lejos del perfil realmente deseado en la competencia digital docente

Esta situación provoca que el desarrollo de la competencia digital en el personal docente sea imprescindible actualmente (Gisbert Cervera et al., 2016), para lo cual se deben presentar programas formativos que permitan tener un profesorado competente digitalmente (Esteve Mon et al., 2014), para un uso educativo adecuado de las diversas tecnologías (Gisbert Cervera et al., 2016).

Tal y como establecen diversos estudios, la formación inicial del profesorado en la utilización de las TIC (Cózar Gutiérrez y Roblizo Colmenero, 2014) puede centrarse en lo puramente técnico (Durán Cuartero et al., 2016) o en el desarrollo de habilidades informacionales (Esteve Mon et al., 2014). Lo ideal es una combinación de ambas (Gisbert Cervera y Lázaro Cantabrana, 2015).

Los instrumentos elaborados para analizar la competencia digital docente centran sus análisis en diversas vertientes. Para Roblizo Colmenero y Cózar Gutiérrez (2015), la competencia digital docente tiene dos ramas claramente definidas: por un lado, las fundamentadas en la tecnología y, por otro lado, en las pedagógicas. En cambio, Lázaro Cantabrana y Gisbert Cervera (2015) establecen una serie de componentes, tales como desarrollar un perfil de necesidades formativas; acceso a información almacenada en entornos virtuales; utilización de las TIC para organizar, interpretar y representar la información; valoración de la información y transmisión de esta (Avello-Martínez y Marín, 2016).

La competencia digital docente está compuesta por 5 áreas competenciales y 21 competencias estructuradas en 6 niveles de conocimientos. Las 5 áreas en las que se dividen son información y alfabetización informacional; comunicación y colaboración; creación de contenidos digitales; seguridad; y resolución de problemas (Instituto Nacional de Tecnologías Educativas y de Formación del Profesorado [INTEF], 2017).

En diversas investigaciones, cuando el estudiantado recibe formación centrada en el área competencial, concretamente en la de información y alfabetización informacional, se considera, en los tiempos actuales, como estudiantado nativo digital, debido al hecho de convivir con la tecnología desde prácticamente su nacimiento (Gewerc y Montero, 2015), por lo que la valoran

Antonio-José Moreno-Guerrero, Nuria Miaja-Chippirraz, Ana Bueno-Pedrero y Laura Borrego-Otero

Los artículos de la Revista Electrónica Educare del Centro de Investigación y Docencia en Educación de la Universidad Nacional, Costa Rica, se comparten bajo términos de la Licencia Creative Commons: Reconocimiento, № Comercial, Sin Obra Derivada 3.0 Costa Rica. Las autorizaciones adicionales a las aquí delimitadas se pueden obtener en el correo: educare@una.cr 
de forma adecuada. Esto se debe, principalmente, a que el área competencial de información y alfabetización permite adquirir habilidades para desarrollar tareas educativas de cualquier materia (Correa Gorospe et al., 2015). El hacer uso de los recursos tecnológicos permiten adquirir una formación que favorece la búsqueda de información en internet, interpretarla, gestionarla, estructurarla, valorarla y evaluarla (Cabezas González et al., 2014). Todo ello, a pesar de la formación académica que reciben para el desarrollo de la competencia digital sea pobre (Roblizo Colmenero y Cózar Gutiérrez, 2015). Este hecho se focaliza, principalmente, en docentes que acceden a la Educación Secundaria (Álvarez y Gisbert Cervera, 2015).

Se determina que el personal docente debe hacer un uso de las dimensiones tecnológica, información, axiológica, pedagógica y comunicativa para promover una formación adecuada, en competencia digital, en sus estudiantes (Rangel Baca, 2015). Debe eliminarse la falsa creencia de que el uso de las TIC se fundamenta en un manejo adecuado y correcto de dispositivos y recursos tecnológicos (Fernández Cárdenas et al., 2015).

\section{Metodología}

El estudio presentado a continuación es de corte cuantitativo, con alcance descriptivo, mediante diseño no experimental, de paradigma longitudinal (Hernández Sampieri et al., 2014). La muestra seleccionada es no probabilística.

La población participante en el estudio está conformada por 153 estudiantes del Máster de Profesorado de Educación Secundaria Obligatoria, Bachillerato, Formación Profesional y Enseñanza de Idiomas en el Campus de Ceuta, de los tres últimos cursos académicos, compuestos por un $23.5 \%$ de estudiantes del curso 2016-2017, un $47.7 \%$ del curso $2017-2018$ y un $28.8 \%$ del curso 2018-2019.

Las características socioeducativas de la muestra implicada en este estudio indican que el $58.8 \%$ son mujeres, mientras que el $41.2 \%$ son hombres. Las edades oscilan entre los 20 y 25 años (50.7\%), entre los 26 y 30 años (24.8\%) y más de 30 años (24.8\%). El 54.9\% de estudiantes tiene una formación previa en la rama de humanidades y ciencias sociales (Filologías, filosofía, ...), y un $45.1 \%$ en la rama científica-tecnológica (matemáticas, biología, ...).

El instrumento aplicado en la investigación es un cuestionario que se fundamenta en el portafolio elaborado por el Instituto Nacional de Tecnologías Educativas y de Formación del Profesorado (INTEF), en el año 2017, concretamente en el mes de octubre. Este fue elaborado por miembros del Ministerio de Educación y Formación Profesional, miembros de delegaciones territoriales de las comunidades autónomas, personas expertas en la materia, docentes de diversas etapas educativas, personal de consultorías y equipos investigadores de universidades desde el año 2012, considerado por ello el instrumento validado mediante la prueba de validez de contenidos (INTEF, 2017). 
http://doi.org/10.15359/ree.24-3.25

http://www.una.ac.cr/educare

educare@una.ac.cr

Se aplicó el coeficiente de consistencia interna alfa (a) de Cronbach (Bernal, 2010) y el área estudiada, denominada información y alfabetización informacional, ha obtenido un valor de $a=.829$, lo cual significa que el instrumento es considerado fiable.

El cuestionario se centra en una de las áreas competenciales establecidas por el INTEF, denominada información y alfabetización informacional, la cual está compuesta por 3 bloques, con un total de 16 ítems, además de las variables sociodemográficas (edad, género, curso académico y especialidad).

El primero de los bloques es navegación, datos y filtrado de información, datos y contenidos digitales en el que se analiza, si el estudiantado es capaz de "buscar información, datos y contenidos digitales en red, y acceder a ellos, expresar de manera organizada las necesidades de información, encontrar información relevante para las tareas docentes" (INTEF, 2017, p. 27). Este bloque está confirmado, a su vez, por 5 ítems.

El segundo bloque, evaluación de información, datos y contenidos digitales trata de identificar en el alumnado su facultad de "reunir, procesar, comprender y evaluar información, datos y contenidos digitales de forma crítica" (INTEF, 2017, p. 19), compuesto por 6 ítems.

Finalmente, el bloque tres, almacenamiento y recuperación de información, datos y contenidos digitales, analiza en el estudiante su habilidad para "gestionar y almacenar información, datos y contenidos digitales para facilitar su recuperación, organizar información, datos y contenidos digitales" (INTEF, 2017, p. 21), conformado por 5 ítems.

La escala de respuesta es de tipo Likert, de 6 puntos, distribuidos en tres bloques de competencias, la primera de ellas, de nivel básico, está compuesta por los niveles A1 y A2; la segunda es nivel intermedio, formada por los niveles B1 y B2, y finalmente, la última dimensión, es nivel avanzado, comprendido por los niveles C1 y C2. Esta categorización permite establecer el nivel que presentan el estudiantado en cada uno de los niveles.

La recogida de los datos se realizó durante los cursos académicos 2016/2017, 2017/2018 y 2018/2019, en los primeros días de clase, con el formulario de Google, a excepción de las personas matriculadas en el curso 2016/2017, a quienes se les presentó el cuestionario durante el mes de noviembre del año 2017.

Los sujetos participantes en el estudio colaboraron en el desarrollo y elaboración del cuestionario. Presentaron una predisposición positiva en todo momento. El tiempo medio de elaboración de la prueba fue aproximadamente de 20 minutos.

\section{Resultados, análisis y discusión}

Los resultados que se presentan a continuación muestran la situación actual en la que se encuentran futuros grupos docentes, describen los perfiles que muestran en el área competencial de información y alfabetización informacional.

6

Antonio-José Moreno-Guerrero, Nuria Miaja-Chippirraz, Ana Bueno-Pedrero y Laura Borrego-Otero

Los artículos de la Revista Electrónica Educare del Centro de Investigación y Docencia en Educación de la Universidad Nacional, Costa Rica, se comparten bajo términos de la Licencia Creative Commons: Reconocimiento, № Comercial, Sin Obra Derivada 3.0 Costa Rica. Las autorizaciones adicionales a las aquí delimitadas se pueden obtener en el correo: educare@una.cr 
http://doi.org/10.15359/ree.24-3.25

La media arrojada por los diversos ítems analizados muestra un nivel medio en competencia digital del estudiantado en los tres bloques (Tabla 1).

La dimensión que presenta valores más altos es la de almacenamiento y recuperación de información, datos y contenidos digitales, mientras donde presentan un nivel más bajo es en la dimensión relacionada con la evaluación de información, datos y contenidos digitales.

Tabla 1: Media del área competencial de información y alfabetización informacional

\begin{tabular}{|c|c|c|}
\hline Bloques de competencias & Ítems & Media \\
\hline \multirow{5}{*}{$\begin{array}{l}\text { Navegación, búsqueda y filtrado de } \\
\text { información, datos y contenidos digitales. }\end{array}$} & Buscar recursos en red & 3.32 \\
\hline & Navegar en internet & 3.38 \\
\hline & Expresar de manera organizada necesidades de información & 3.5 \\
\hline & Buscadores de internet & 2.62 \\
\hline & Estrategias de búsqueda de información & 3.41 \\
\hline \multirow{6}{*}{$\begin{array}{l}\text { Evaluación de información, datos y } \\
\text { contenidos digitales }\end{array}$} & Recursos educativos & 2.48 \\
\hline & Identificación de recursos & 2.94 \\
\hline & Selección de información & 3.14 \\
\hline & Selección de recursos educativos & 3.17 \\
\hline & Búsqueda de recursos educativos & 2.63 \\
\hline & Recopilación de información & 2.96 \\
\hline \multirow{5}{*}{$\begin{array}{l}\text { Almacenamiento y recuperación } \\
\text { de información, datos y contenidos } \\
\text { digitales }\end{array}$} & Sistema de almacenamiento & 3.40 \\
\hline & Organizar y guardar la información & 3.24 \\
\hline & Almacenamiento de contenidos educativo & 3.01 \\
\hline & Organización de la información & 3.63 \\
\hline & Seguridad de la información & 3.73 \\
\hline
\end{tabular}

Nota: Elaboración propia.

Centrando el análisis en los bloques de competencias que componen esta área, recogidos en la Tabla 2, se pueden observar que, en relación con la navegación, búsqueda y filtrado de información, datos y contenidos digitales, los niveles se sitúan entre A2 y B2, dependiendo del ítem. En cuanto a la búsqueda de recursos en red, el profesorado presenta un nivel A2, por lo que hacen uso de la red para buscar recursos y herramientas de perfil didáctico, sin hacer búsqueda avanzada o especializada. A la hora de navegar por la red, su nivel medio se sitúa en B1, en donde es capaz de realizar búsquedas en internet para localizar recursos y datos relacionados con la docencia en distintos formatos, sin centrar su búsqueda en el primer recurso que encuentra.

A la hora de expresar, de manera organizada, determinadas necesidades de información, su nivel medio también se sitúa en un B1, esto es, resulta capaz de orientar al alumnado para realizar determinadas búsquedas de información en la red, les ofrece determinados recursos que les permitan encontrar la información que necesitan, aunque no realizan antes una recopilación de recursos. 
http://doi.org/10.15359/ree.24-3.25

http://www.una.ac.cr/educare

educare@una.ac.cr

Respecto a los buscadores de internet, el nivel medio también se sitúa en B1: el personal docente tiene el conocimiento de determinados sitios web o repositorios oficiales donde el alumnado puede encontrar información relevante asociada a aspectos educativos, además de plataformas virtuales que favorecen el aprendizaje colaborativo, aunque en este caso no ofrece recursos propios elaborado por ella o él mismo.

Finalmente, en las estrategias de búsqueda de información, el nivel se sitúa por encima del resto de ítems, donde B2 es el perfil de la población analizada. En este caso, el profesorado es capaz de realizar búsqueda por palabras clave, tanto en castellano como en otro idioma, para encontrar elementos pedagógicos que favorezcan el proceso de enseñanza y aprendizaje.

Al observar lo datos se puede determinar que el nivel C1 y C2 presenta valores muy bajos con respecto al resto de niveles, donde los datos no superan el 16\% del total de la población en ninguno de los casos.

Tabla 2: Navegación, búsqueda y filtrado de información, datos y contenidos digitales. Área de información y alfabetización informacional

\begin{tabular}{|c|c|c|c|c|c|c|c|}
\hline \multirow[t]{2}{*}{ Ítems } & \multicolumn{6}{|c|}{ Niveles competenciales progresivos de manejo } & \\
\hline & A1 & $\mathrm{A} 2$ & B1 & B2 & $\mathrm{C} 1$ & $\mathrm{C} 2$ & \\
\hline \multirow{2}{*}{ Buscar recursos en red } & 2 & 56 & 27 & 37 & 21 & 10 & $\mathrm{~N}$ \\
\hline & 1.3 & 36.6 & 17.6 & 24.2 & 13.7 & 6.5 & $\%$ \\
\hline \multirow{2}{*}{ Navegar en internet } & 28 & 6 & 61 & 20 & 14 & 24 & $\mathrm{~N}$ \\
\hline & 18.3 & 3.9 & 39.9 & 13.1 & 9.2 & 15.7 & $\%$ \\
\hline \multirow{2}{*}{$\begin{array}{l}\text { Expresar de manera organizada } \\
\text { necesidades de información }\end{array}$} & 8 & 26 & 49 & 35 & 21 & 14 & $\mathrm{~N}$ \\
\hline & 5.2 & 17 & 32 & 22.9 & 13.7 & 9.2 & $\%$ \\
\hline \multirow{2}{*}{ Buscadores de internet } & 26 & 47 & 53 & 19 & 2 & 6 & $\mathrm{~N}$ \\
\hline & 17 & 30.7 & 34.6 & 12.4 & 1.3 & 3.9 & $\%$ \\
\hline \multirow{2}{*}{$\begin{array}{l}\text { Estrategias de búsqueda de } \\
\text { información }\end{array}$} & 7 & 22 & 49 & 58 & 10 & 7 & $\mathrm{~N}$ \\
\hline & 4.6 & 14.4 & 32 & 37.9 & 6.5 & 4.6 & $\%$ \\
\hline
\end{tabular}

Nota: Elaboración propia.

Según lo marcado en la Tabla 3, sobre la evaluación de información, datos y contenidos digitales, la tendencia es similar a los datos ofrecidos en la Tabla 2, donde el perfil del personal docente se sitúa entre $A 2$ y B2. En relación con los recursos educativos, el profesorado presenta un nivel medio de $\mathrm{A} 2$, esto es, es capaz de analizar la integración de los recursos pedagógicos a los elementos propios del currículo, se centrado principalmente en la programación didáctica, no llega a ser establecer criterios de calidad de los recursos educativos disponibles en internet.

Sobre la identificación de recursos el nivel medio se sitúa en B1, aunque con resultados muy parejos en el nivel competencial B2, es decir, es capaz de replantearse si los recursos educativos encontrados son adecuados o no para el grupo clase al que va destinado; llega en algunos casos a evaluarlos, para ver si se relacionan con lo establecido en la programación didáctica. 
http://doi.org/10.15359/ree.24-3.25

Con respecto a la selección de información, el nivel competencial del futuro personal docentes se sitúa en B1: capaz de, entre varias opciones educativas, seleccionar la más adecuada a las necesidades reales de sus discentes.

En relación con la selección de recursos educativos, su nivel es superior a lo marcado en la media, situándose su perfil competencial en B2: es capaz de analizar y valorar las experiencias educativas ofrecidas por otros grupos docentes $u$ otros centros educativos, para adecuarlas a su propia práctica docente.

A la hora de buscar recursos educativos, el nivel competencial del personal docente se sitúa en B1: es capaz de comparar, contrastar e integrar los recursos e información encontrada en la red de forma continua, analizando constantemente su utilidad en cada momento del proceso de enseñanza y aprendizaje.

Finalmente, en la recopilación de información, el nivel competencial se sitúa en B: presenta la capacidad de desarrollar estrategias propias de búsqueda de información y recursos pedagógicos relevantes para la práctica docente.

Como ocurría en el anterior bloque de competencias, los niveles superiores de competencia digital docente, centrados en C1 y C2, no superan el 17\% en ninguno de los casos.

Tabla 3: Evaluación de información, datos y contenidos digitales. Área de información y alfabetización informacional

\begin{tabular}{|c|c|c|c|c|c|c|c|}
\hline \multirow[t]{2}{*}{ Ítems } & \multicolumn{6}{|c|}{ Niveles competenciales progresivos de manejo } & \\
\hline & $\mathrm{A} 1$ & A2 & B1 & B2 & $\mathrm{C} 1$ & $\mathrm{C} 2$ & \\
\hline \multirow{2}{*}{ Recursos educativos } & 40 & 58 & 16 & 24 & 11 & 4 & $\mathrm{~N}$ \\
\hline & 26.1 & 37.9 & 10.5 & 15.7 & 7.2 & 2.6 & $\%$ \\
\hline \multirow{2}{*}{ Identificación de recursos } & 29 & 25 & 44 & 41 & 9 & 5 & $\mathrm{~N}$ \\
\hline & 19 & 16.3 & 28.8 & 26.8 & 5.9 & 3.3 & $\%$ \\
\hline \multirow{2}{*}{ Selección de información } & 28 & 6 & 65 & 34 & 10 & 10 & $\mathrm{~N}$ \\
\hline & 18.3 & 3.9 & 42.5 & 22.2 & 6.5 & 6.5 & $\%$ \\
\hline \multirow{2}{*}{ Selección de recursos educativos } & 24 & 33 & 27 & 39 & 22 & 8 & $\mathrm{~N}$ \\
\hline & 15.7 & 21.6 & 17.6 & 25.5 & 14.4 & 5.2 & $\%$ \\
\hline \multirow{2}{*}{ Búsqueda de recursos educativos } & 46 & 22 & 51 & 15 & 14 & 5 & $\mathrm{~N}$ \\
\hline & 30.1 & 14.4 & 33.3 & 9.8 & 9.2 & 3.3 & $\%$ \\
\hline \multirow{2}{*}{ Recopilación de información } & 27 & 45 & 29 & 28 & 7 & 17 & $\mathrm{~N}$ \\
\hline & 17.6 & 29.4 & 19 & 18.3 & 4.6 & 11.1 & $\%$ \\
\hline
\end{tabular}

Nota: Elaboración propia. 
http://doi.org/10.15359/ree.24-3.25

http://www.una.ac.cr/educare

educare@una.ac.cr

Finalmente, y según los datos ofrecidos en la Tabla 4, sobre la capacidad de almacenamiento y recuperación de información, datos y contenidos digitales, observamos que el abanico se amplía situándose los niveles competenciales del profesorado entre A2 y C2, es decir, un resultado con mayor dispersión en las respuestas, lo cual rompe con la tendencia marcada en los dos bloques anteriores. En relación con el sistema de almacenamiento, los resultados son parejos. Se sitúan en el pico más alto en el nivel A2, donde el profesorado entiende cómo se almacena la información en distintos dispositivos, y hace uso de ellos cuando así lo requiere.

Tabla 4: Almacenamiento y recuperación de información, datos y contenidos digitales. Área de información y alfabetización informacional

\begin{tabular}{|c|c|c|c|c|c|c|c|}
\hline \multirow{2}{*}{ Ítems } & \multicolumn{6}{|c|}{ Niveles competenciales progresivos de manejo } & \\
\hline & $\mathrm{A} 1$ & $\mathrm{~A} 2$ & B1 & B2 & $\mathrm{C} 1$ & $\mathrm{C} 2$ & \\
\hline \multirow{2}{*}{ Sistema de almacenamiento } & 28 & 33 & 18 & 24 & 24 & 26 & $\mathrm{~N}$ \\
\hline & 18.3 & 21.6 & 11.8 & 15.7 & 15.7 & 17 & $\%$ \\
\hline \multirow{2}{*}{ Organizar y guardar la información } & 28 & 37 & 18 & 27 & 27 & 16 & $\mathrm{~N}$ \\
\hline & 18.3 & 24.2 & 11.8 & 17.6 & 17.6 & 10.5 & $\%$ \\
\hline \multirow{2}{*}{ Almacenamiento de contenidos educativos } & 14 & 56 & 26 & 37 & 11 & 9 & $\mathrm{~N}$ \\
\hline & 9.2 & 36.6 & 17 & 24.2 & 7.2 & 5.9 & $\%$ \\
\hline \multirow{2}{*}{ Organización de la información } & 5 & 14 & 67 & 23 & 34 & 10 & $\mathrm{~N}$ \\
\hline & 3.3 & 9.2 & 43.8 & 15 & 22.2 & 6.5 & $\%$ \\
\hline \multirow{2}{*}{ Seguridad de la información } & 17 & 37 & 14 & 24 & 24 & 37 & $\mathrm{~N}$ \\
\hline & 11.1 & 24.2 & 9.2 & 15.7 & 15.7 & 24.2 & $\%$ \\
\hline
\end{tabular}

Nota: Elaboración propia.

Con respecto a organizar y guardar la información ocurre algo similar al anterior ítem, aunque en este caso, el pico de respuesta es ligeramente superior al resto, pues se ubica en el nivel A2: los docentes y las docentes conocen la existencia de herramientas y recursos en línea para guardar y organizar la información en distintos formatos.

Sobre el almacenamiento de contenidos educativos, el nivel competencial del profesorado está en A2: es capaz de almacenar y organizar los recursos educativos para ponerlos en práctica en los procesos de enseñanza y aprendizaje.

En cuanto a la organización de la información, el nivel competencial se sitúa en B1. En este caso, los futuros grupos docentes son capaces de compartir distintos tipos de archivos mediante correo electrónico, pero no son capaces de hacerlo por otros medios. 
http://doi.org/10.15359/ree.24-3.25

Sobre la seguridad de la información, el nivel competencial se sitúa en C2, es el más alto de los niveles alcanzados por una parte del estudiantado: es capaz de combinar distintos medios y recursos para transferir archivos pedagógicos entre dispositivos para uso personal.

\section{Conclusiones}

Para que el profesorado sea competente digitalmente en el área de información y alfabetización informacional, debe tener una serie de aptitudes que le permitan transmitirlas adecuadamente al alumnado, para fomentar en él la competencia clave, denominada competencia digital, recogida de manera general, en el contexto español, en la Ley Orgánica 2/2006 (Jefatura del Estado, 2006) de Educación, modificada por la Ley Orgánica 8/2013 (Jefatura del Estado, 2013) para la mejora de la calidad educativa, y forma específica en la Orden 65/2015 (Jefatura del Estado, 2015) del actual sistema educativo español.

De manera más específica, el personal docente, para navegar, buscar y filtrar información, datos y contenidos digitales, debe ser capaz de utilizar estrategias de búsqueda y filtrado de información para desarrollar buenas prácticas. Además, debe incorporar las últimas tendencias educativas, seleccionar y adaptar los recursos pedagógicos a las necesidades del alumnado y del currículo; ser capaz de identificar recursos educativos y buenas prácticas en la red para incorporar en el proceso de enseñanza y aprendizaje; elaborary participar en una red de docentes, intercambiar experiencias y recursos para incorporar en la práctica docente; y finalmente, buscar y filtrar todos los recursos pedagógicos existentes en las diversas comunidades docentes (INTEF, 2017 en consonancia a lo indicado por Avello-Martínez y Marín, 2016).

Todo ello dista de los resultados dados en la presente investigación, donde el profesorado realiza búsqueda de recursos didácticos, pero sin criterios avanzados, aunque no escoge el primer recurso que encuentra; puede orientar al alumnado sobre la búsqueda de determinados recursos o repositorios educativos, pero sin haberlos analizado previamente y sin haberlos elaborado; además de realizar búsqueda en diversos idiomas. Lo indicado sigue la tónica establecida por Roblizo Colmenero y Cózar Gutiérrez (2015), quienes establecen que la formación docentes en esta área es media-baja en los distintos bloques de competencia.

A rasgos generales, en relación con la evaluación de información, datos y contenidos digitales, el personal docente debe saber formar a otros grupos de docentes sobre la calidad de los recursos educativos; interactuar con profesionales sobre intereses educativos propios y valorar de manera crítica; mantener una actitud crítica con fuentes de información y perfiles profesionales relacionados con los procesos de enseñanza y aprendizaje y transmitir esa actitud al alumnado; combinar recursos tecnológicos y no tecnológicos para evaluar la información educativa; establecer licencias adecuadas cuando comparte recursos educativos; y enseñar al alumnado cómo localizar información, evaluarla y combinarla con otras fuentes de información 
http://doi.org/10.15359/ree.24-3.25

http://www.una.ac.cr/educare

educare@una.ac.cr

para generar su propio contenido (INTEF, 2017). De forma más específica, en dicho documento se indican, de manera específica, cada uno de los niveles en cada uno de los ítems que componen los diversos bloques de competencia de esta área competencial.

En la misma tónica que el anterior bloque de competencias, existen diferencias significativas a lo establecido en el anterior párrafo, dado que el personal docente incorpora recursos educativos a los procesos de enseñanza y aprendizaje, y valora si son adecuados o no para los estudiante, aunque sin establecer criterios claros sobre su calidad; analiza prácticas docentes para adecuarlas a los procesos de enseñanza y aprendizaje; contrasta y compara recursos educativos encontrados en la red para incluirlos en la práctica docente, y establece criterios propios de búsqueda de información.

Con respecto al almacenamiento y recuperación de información, datos y contenidos digitales, el equipo de docentes debe disponer de servicios de almacenamiento de contenidos educativos on-line y compartirlos con sus estudiantes; establecer una estrategia de almacenado adecuada y coherente; generar repositorios educativos y compartirlos con estudiantes y docentes; seleccionar información relevante a nivel educativo y compartirla; finalmente, combinar diferentes formar de conexión para transferir archivos (INTEF, 2017).

Esta área sigue la línea marcada por las otras dos, en la que el personal docente comprende cómo se almacena la información en distintos dispositivos, mediante soporte físico o en línea; transmiten la documentación mediante correo electrónico. Al respecto, esto está en contraposición con lo marcado por Cantón et al. (2017), quienes consideran que presentan una formación adecuada en lo que a comunicación se refiere.

Como podemos observar, el personal docente muestra un nivel medio-bajo en el área competencial de información y alfabetización informacional. Al respecto, se coincide con lo establecido por Cabezas González et al. (2014), sobre que es poco probable que puedan transmitir y formar de manera adecuada sobre la competencia digital docente.

Por ello, las instituciones universitarias centradas en la formación de futuro profesorado, sobre todo focalizados en este estudio (Educación Secundaria Obligatoria, Formación Profesional, Bachillerato, Escuela Oficial de Idiomas y otras enseñanzas no obligatorias), deben plantearse el desarrollo de la competencia digital docente, concretamente en el área de información y alfabetización informacional, ya sea mediante materias específicas que así lo permitan o mediante la enseñanza transversal en las distintas materias de la oferta formativa, para potenciar y reforzar la formación de las distintas áreas competenciales que conforman la competencia digital docente.

La principal dificultad encontrada en la investigación fue presentar el cuestionario sobre competencia digital docente con todas las áreas de competencia digital. Observamos que el 
http://doi.org/10.15359/ree.24-3.25

personal docente que participó en el ensayo piloto decía que era muy denso y que se cansaba en su desarrollo, por ello, pasamos a realizar los cuestionarios por áreas, y enviándolos de manera escalonada, para evitar el cansancio y el hecho de dejar de responder a la mitad.

De cara a futuras investigaciones, habría que plantearse varias situaciones. Analizar la competencia digital docente del profesorado universitario para determinar si es capaz de desarrollar su enseñanza de manera transversal. Otra posibilidad es analizar el resto de áreas competenciales, para saber si los niveles son semejantes a los datos arrojados en esta investigación, además de permitir establecer un plan de formación más específico y adecuado a las necesidades reales de la población a la que va dirigida. Finalmente, se puede plantear el análisis correlacional de los datos ofrecidos con el género, la edad y la rama de conocimientos del estudiantado.

\section{Declaración de Material complementario}

Este artículo tiene disponible como material complementario:

- La versión preprint del artículo en https://doi.org/10.5281/zenodo.3344532

\section{Referencias}

Agreda Montoro, M., Hinojo Lucena, M. A. y Sola Reche, J. M. (2016). Diseño y validación de un instrumento para evaluar la competencia digital de los docentes en la educación superior española. Pixel-Bit. Revista de Medios y Educación, 49, 39-56. https://doi.org/10.12795/ pixelbit.2016.i49.03

Álvarez, J.-F. y Gisbert Cervera, M. (2015). Grado de alfabetización informacional del profesorado de secundaria en España: Creencias y autopercepciones. Comunicar, 23(45), 187-194. https://doi.org/10.3916/C45-2015-20

Avello-Martínez, R. y Marín, V. I. (2016). La necesaria formación de los docentes en aprendizaje colaborativo. Profesorado. Revista de currículum y formación del profesorado, 20(3), 687-713. https://recyt.fecyt.es/index.php/profesorado/article/view/54603

Bernal, C. A. (2010). Metodología de la investigación. Administración, economía, humanidades y ciencias sociales (3. ${ }^{\mathrm{a}}$ ed.). Pearson Education.

Cabezas González, M., Casillas Martín, S. y Pinto Llorente, A. M. (2014). Percepción de los alumnos de educación primaria de la Universidad de Salamanca sobre su competencia digital. EDUTEC. Revista Electrónica de Tecnología Educativa, 48, 1-14. http://www.edutec. es/revista/index.php/edutec-e/article/view/156 
http://doi.org/10.15359/ree.24-3.25

http://www.una.ac.cr/educare

educare@una.ac.cr

Cantón-Mayo, l., Cañón-Rodríguez, R. y Grande-de-Prado, M. (2017). La comunicación como subdimensión de la competencia digital en futuros maestros de primaria. Pixel-Bit. Revista de Medios y Educación, 50, 33-47. https://doi.org/10.12795/pixelbit.2017.i50.02

Correa Gorospe, J. M., Fernández Olaskoaga, L., Gutiérrez-Cabello Barragán, A., Losada Iglesias, D. y Ochoa-Aizpurua Aguirre, B (2015). Formación del profesorado, tecnología educativa e identidad docente digital. Revista Latinoamericana de Tecnología Educativa, 14(1), 45-56. https://doi.org/10.17398/1695-288X.14.1.45

Cózar Gutiérrez, R. y Roblizo Colmenero, M. J. (2014). La competencia digital en la formación de los futuros maestros: Percepciones de los alumnos de los Grados de Maestro de la Facultad de Educación de Albacete. RELATEC. Revista Latinoamericana de Tecnología Educativa, 13(2), 119-133. https://doi.org/10.17398/1695-288X.13.2.119

Durán Cuartero, M., Gutiérrez Porlán, I. y Prendes Espinosa, M. P. (2016). Análisis conceptual de modelos de competencia digital del profesorado universitario. RELATEC. Revista Latinoamericana de Tecnología Educativa, 15(1), 97-114. https://doi.org/10.17398/1695288X.15.1.97

Esteve Mon, F. M., Adell Segura, J. y Gisbert, Cervera, M. (2014). Diseño de un entorno 3D para el desarrollo de la competencia digital en estudiantes universitarios: Usabilidad, adecuación y percepción de utilidad. RELATEC. Revista Latinoamericana de Tecnología Educativa, 13(2), 35-47. https://doi.org/10.17398/1695-288X.13.2.35

Esteve-Mon, F., Gisbert-Cervera, M. y Lázaro-Cantabrana, J. L. (2016). La competencia digital de los futuros docentes: ¿Cómo se ven los actuales estudiantes de educación? Perspectiva educacional. Formación de Profesores, 55(2), 38-54. https://doi.org/10.4151/07189729Vol.55-Iss.2-Art.412

Fernández Martín, F. D., Hinojo Lucena, F. J. y Aznar Díaz, I. (2002). Las actitudes de los docentes hacia la formación en tecnologías de la información y comunicación (TIC) aplicadas a la educación. Contextos Educativos, 5, 253-270. https://doi.org/10.18172/con.516

Fernández Cárdenas, J. M., Yáñez Figueroa, J. A. y Muñoz Villarreal, E. K. (2015). Prácticas morales y normas de netiqueta en las interacciones virtuales de los estudiantes de educación secundaria. Innovación Educativa, 15(69), 57-72. http://www.scielo.org.mx/pdf/ie/v15n69/ v15n69a5.pdf

Gewerc, A. y Montero, L. (2015). Conocimiento profesional y competencia digital en la formación del profesorado. El caso del grado de maestro en educación primaria. RELATEC. Revista Latinoamericana de Tecnología Educativa, 14(1), 31-43. https://doi.org/10.17398/1695$\underline{288 X .14 .1 .31}$ 
Gisbert Cervera, M., González Martínez, J. y Esteve Mon, F. (2016). Competencia digital y competencia digital docente: Una panorámica sobre el estado de la cuestión. Revista Interuniversitaria de Investigación en Tecnología Educativa (RIITE), 0, 74-83. https://doi. org/10.6018/riite2016/257631

Gisbert Cervera, M. y Lázaro Cantabrana, J. L. (2015). Professional development in teacher digital competence and improving school quality from the teacher perspective: A case study. New Approachers in Educational Research, 4(2), 115-122. https://doi.org/10.7821/ naer.2015.7.123

Hernández Sampieri, R., Fernández Collado, C. y Baptista Lucio, M. P. (2014). Metodología de la investigación (6. ${ }^{\mathrm{a}}$ ed.). McGraw-Hill Education.

Instituto Nacional de Tecnologías Educativas y de Formación del Profesorado. (2017). Marco común de competencia digital docente - Septiembre 2017. https://aprende.intef.es/sites/ default/files/2018-05/2017 1020 Marco-Com\%C3\%BAn-de-Competencia-DigitalDocente.pdf

Jefatura del Estado. (4 de mayo de 2006). Ley Orgánica 2/2006, de 3 de mayo, de Educación. Boletín Oficial del Estado, núm. 106-7899, pp. 17158-17207. https://www.boe.es/buscar/ pdf/2006/BOE-A-2006-7899-consolidado.pdf

Jefatura del Estado. (10 de diciembre de 2013). Ley Orgánica 8/2013, de 9 de diciembre, para la mejora de la calidad educativa. Boletín Oficial del Estado, núm. 295-12886, pp. 9785897921. https://www.boe.es/buscar/act.php?id=BOE-A-2013-12886

Jefatura del Estado. (29 de enero de 2015). Orden ECD/65/2015, de 21 de enero, por la que se describen las relaciones entre las competencias, los contenidos y los criterios de evaluación de la educación primaria, a educación secundaria obligatoria y el bachillerato. Boletín Oficial del Estado, núm. 25-738, pp. 6986-7003. https://www.boe.es/buscar/doc. php?id=BOE-A-2015-738

Lázaro Cantabrana, J. L. y Gisbert Cervera, M. (2015). EL desarrollo de la competencia digital docente a partir de una experiencia piloto de formación en alternancia en el Grado de Educación. Educar, 51(2), 321-348. https://doi.org/10.5565/rev/educar.725

Moreno Guerrero, A. J. y López Belmonte, J. (2018). B-Learning teaching method for the development of the teaching and learning process: An experience in the subject educational processes and contexts of the master's degree in teaching compulsory secondary and upper secondary school, vocational training. Proceedings, 2(21), 1-4. https://doi.org/10.3390/proceedings2211358 
http://doi.org/10.15359/ree.24-3.25

http://www.una.ac.cr/educare

educare@una.ac.cr

Rangel Baca, A. (2015). Competencias docentes digitales: Propuesta de un perfil. Pixel-Bit. Revista de Medios y Educación, 46, 235-248. https://doi.org/10.12795/pixelbit.2015.i46.15

Roblizo Colmenero, M. J. y Cózar Gutiérrez, R. (2015). Usos y competencias en TIC en los futuros maestros de educación infantil y primaria: Hacia una alfabetización tecnológica real para docentes. Pixel-Bit. Revista de Medios y Educación, 47, 23-39. https://doi.org/10.12795/ pixelbit.2015.i47.02

Sánchez-Antolín, P., Ramos, F. J. y Sánchez Santamaría, J. (2014). Formación continua y competencia digital docente: El caso de la comunidad de Madrid. Revista lberoamericana de Educación, 65, 91-110. https://doi.org/10.35362/rie650395

Trujillo Torres, J. M. y Raso Sánchez, F. (2010). Formación inicial docente y competencia digital en la convergencia europea (EEES). Enseñanza \& Teaching, 28(1), 49-77. http://campus.usal. es/ revistas trabajo/index.php/0212-5374/article/viewFile/7481/7946

Valverde Berrocoso, J. (2015). La formación universitaria en tecnología educativa: Enfoques, perspectivas e innovación. Revista Latinoamericana de Tecnología Educativa, 14(1), 11-16. https://relatec.unex.es/issue/view/197 